\title{
Neurological immunotherapy in the era of COVID-19 - looking for consensus in the literature
}

Catharina Korsukewitz ${ }^{1}$, Stephen W. Reddel ${ }^{2}$, Amit Bar-Or ${ }^{3}$ and Heinz WiendI ${ }^{1 凶}$

Abstract | The coronavirus disease 2019 (COVID-19) pandemic is concerning for patients with neuroimmunological diseases who are receiving immunotherapy. Uncertainty remains about whether immunotherapies increase the risk of infection with severe acute respiratory syndrome coronavirus 2 (SARS-CoV-2) or increase the risk of severe disease and death upon infection. National and international societies have developed guidelines and statements, but consensus does not exist in several areas. In this Review, we attempt to clarify where consensus exists and where uncertainty remains to inform management approaches based on the first principles of neuroimmunology. We identified key questions that have been addressed in the literature and collated the recommendations to generate a consensus calculation in a Delphi-like approach to summarize the information. We summarize the international recommendations, discuss them in light of the first available data from patients with COVID-19 receiving immunotherapy and provide an overview of management approaches in the COVID-19 era. We stress the principles of medicine in general and neuroimmunology in particular because, although the risk of viral infection has become more relevant, most of the considerations apply to the general management of neurological immunotherapy. We also give special consideration to immunosuppressive treatment and cell-depleting therapies that might increase susceptibility to SARS-CoV-2 infection but reduce the risk of severe COVID-19.

Coronavirus disease 2019 (COVID-19) is a novel illness caused by infection with severe acute respiratory syndrome coronavirus 2 (SARS-CoV-2) ${ }^{1,2}$. The first infections were reported in China in late 2019, and the WHO declared a global pandemic on 11 March 2020. The disease course is highly heterogeneous, ranging from infectious but asymptomatic to severe disease and death, often due to respiratory failure ${ }^{3}$. The host reaction to the virus can include antibody-mediated inflammation ${ }^{4}$ and a cytokine storm ${ }^{5}$ that are thought to have a major impact on outcome. Most people recover with only supportive care, indicating that the infection induces short-term immunity, but whether long-term immunity develops or recurrent circulation of SARS-CoV-2 can be expected - as seen with other coronaviruses - is unknown ${ }^{6}$.

The COVID-19 pandemic raises important questions about the risk to patients with neuroimmunological diseases who are treated with immunotherapies. These diseases - a key example of which is multiple sclerosis (MS) - are thought to result from immune tolerance dysfunction that affects immune regulatory networks ${ }^{7}$ and often necessitates continuous immunosuppressive or immunomodulatory therapy. Immunosuppressive therapies can limit immune competence, whereas immunomodulatory therapies adjust the immune system without affecting immune competence ${ }^{8}$. Immunotherapies can affect the risk of infections ${ }^{9}$ and some therapies are associated with an increased risk from particular types of pathogens $^{10}$.

Several elements of the risk of COVID-19 in patients with neuroimmunological disease are unclear; for example, whether these patients have an increased risk of being infected by SARS-CoV-2 after exposure, whether they have an increased risk of developing a clinical rather than asymptomatic infection, and whether they have an increased risk of a clinical infection becoming severe enough to require intensive care or to lead to persistent disability or death. We refer to the sum of these risks as an 'increased risk of severe COVID-19'. A further complication is that there is a rationale for immunotherapy being beneficial in COVID-19, especially in the management of complications such as acute respiratory distress syndrome (ARDS) $)^{11,12}$, though caution is needed given 


\section{Key points}

- The risk that the coronavirus disease 2019 (COVID-19) pandemic poses for people who are receiving immunotherapy for neuroimmunological disease remains unclear.

- Guidelines and statements have been published by societies and individuals, but the level of consensus differs for different aspects; we use a Delphi-like process to clarify where consensus exists.

- Without evidence, management of neuroimmunological diseases in the context of COVID-19 requires application of the first principles of immunotherapy, taking into account disease-related, patient-related, physician-related, environment-related and COVID-19-related factors.

- In general, corticosteroids, intravenous immunoglobulin and/or plasma exchange for the treatment of acute neuroimmunological deteriorations can be administered with low risk in the COVID-19 pandemic.

- In general, ongoing immunotherapy should not be stopped because of the COVID-19 pandemic; treatment initiation and optimization are also recommended.

- For some aspects of immunotherapy in the context of COVID-19, consensus in the literature is low, and collection of data in patient registries is important for resolving these uncertainties. small effects can be excluded. For example, in a trial in which population $\mathrm{X}$ receives intervention $\mathrm{Y}$ that increases the likelihood of outcome $\mathrm{Z}$ from $2 \%$ to $4 \%$, a sample size of 1,141 per group is needed for an $\alpha$ of 0.05 , a $\beta$ of 0.2 and a power of 0.8 . In this scenario, $\mathrm{X}$ represents patients with neuroimmunological disease, Y represents the patients' immunotherapy and $\mathrm{Z}$ is the likelihood of requiring intensive care or of death if the drug doubles the risk of catastrophic COVID-19. We simply do not have that amount of data yet - until we do, we should work from first principles.

In this scenario an explicit reiteration of the first principles of medicine is warranted in balancing neuroimmune treatments with the risk of COVID-19 (BOX 2), including pertinent factors that influence decision making in addition to the recommendations (BOX 3; TABLE 1). The following points and factors are critical for making decisions about the initiation, continuation and optimization of a patient's neuroimmunological treatment:

the negative history of immunotherapy for infection, including negative trials of corticosteroids for ARDS ${ }^{13}$. Initial reports of patients with immunosuppression and COVID-19 are complicated but suggest no general deleterious effect ${ }^{14}$ and a potentially helpful role of immunosuppression, especially in patients who are at risk of serious complications in the second phase of the disease ${ }^{15,16}$.

Several national and international guidelines, recommendations and statements and individual opinion pieces have addressed the management of patients with neuroimmunological disease, especially MS, during the COVID-19 pandemic. However, these publications are heterogeneous in their level of detail and their findings. In this Review, we bring together the existing information and develop an overview of the current consensus in the literature based on a 'Delphi-like' process ${ }^{17}$ (BOX 1). We searched the available literature, including publications of national and international societies and statements related to COVID-19 and neurology (Supplementary Table 1). We identified key questions addressed within these publications and compiled the answers, indicating the level of consensus in the literature (TABLE 1). On this basis, we propose an algorithm that encompasses the factors that influence decision-making for patients with neuroimmunological diseases in the COVID-19 era.

\section{The challenges of COVID-19}

In the modern medical era of randomized controlled trials, Cochrane analyses and, at the very least, detailed series, we are habituated to making decisions supported by an evidence base. In the unusual situation of the COVID-19 pandemic, a potentially lethal novel construct has been superimposed on the existing complexities of our patients and their management, and little specific evidence is available, making management decisions difficult and complex.

In multiple series published to date, a total of several hundreds of patients receiving immunosuppressive therapy for various diseases have recovered from COVID-19, thereby reassuring us that the infection is not necessarily catastrophic. Nevertheless, we must keep in mind that very large numbers of patients must be studied before
- First, do no harm - inactivity that leads to permanent damage is a form of harm by neglect, but caution should be exercised if the situation allows (for example, if the patients have highly relevant comorbidities, or if the burden of the underlying disease is low).

- In general, immunosuppressive strategies produce a substantial benefit in relation to the disease being treated, but the trade-off is often a modest increase in the risk of infection that is dependent on dose, duration and intensity of therapy.

- Disease-related factors; for example, how serious and reversible the patient's disease is (for example, myasthenia is reversible, so undertreatment is more acceptable than for aquaporin 4-related neuromyelitis optica, which is not), the individual's clinical course and the current disease activity.

- Patient-related factors; for example, the factors that influence the patient's risk of serious or lethal COVID-19 (including age, sex, comorbidities and perception of the situation, such as risk aversion).

- The patient should be asked whether the disease they have or the one they could get matters more to them - the doctor's risk prioritization does not always match the patient's.

- Physician-related factors, such as experience, understanding of the disease and its treatment, treatments available and monitoring capabilities. lihood of exposure to COVID-19 now and in the future, and local logistical issues, such as limitations in patient support, infusion units or intensive care units.

- Treatment-related factors, such as the extent to which treatment could affect the response to COVID-19, whether the treatment is reversible and whether the dose can be reduced or deferred.

\section{Treatment of acute deteriorations}

Acute deteriorations or relapses of neuroimmunological diseases are usually treated with corticosteroid pulse therapy, plasma exchange or intravenous immunoglobulin (IVIg) before initiation of or changes to long-term disease-modifying therapies (DMTs). In this section, we
- Environmental factors; for example, the local like- 
discuss the use of these acute treatments in the context of COVID-19.

\section{Corticosteroids}

Corticosteroids dampen cytokine responses and the recruitment of leukocytes, and suppress $\mathrm{T}$ cell activation and differentiation ${ }^{18}$; for these reasons, they can increase the risk of infection as a result of short-term lymphopenia ${ }^{19}$. However, short-term therapy is not usually associated with a marked increase in the risk of infection ${ }^{20}$. Chronic, high doses of steroids increase the rate of infections and are associated with other long-term adverse effects, such as hypertension, osteoporosis and muscle weakness.

The question of short-term corticosteroid therapy for acute relapses of neuroimmunological diseases in the COVID-19 era was not addressed in all sources included in our Delphi-like analysis. Where this question was addressed, it related to MS, and we identified agreement that steroid pulse therapy should be administered for a relapse with a relevant neurological deficit (TABLE 1, Question 2). For relapses with very minor symptoms (for example, dysaesthesias) or where no evidence indicates a benefit of treatment (for example, repetitive pulses in progressive disease), corticosteroids should currently be avoided. Steroid tapering - which is sometimes done to prolong and consolidate the pulse effect when pulse treatment has not produced substantial clinical improvement - is not recommended. Overall, short-term treatment with corticosteroids carries a low risk in relation to COVID-19 and should be administered whenever necessary.

Corticosteroid therapy has been used in the treatment of SARS-associated and Middle East respiratory syndrome (MERS)-associated pneumonia ${ }^{21}$, but analysis has revealed no beneficial effects and, in some cases, harmful effects ${ }^{22}$. Evidence in relation to the use of corticosteroids for the treatment of COVID-19 is lacking and expert opinions are contradictory - their use has been recommended for severe COVID-19 when the criteria for ARDS is fulfilled, but not for severe COVID-19 without $\mathrm{ARDS}^{23}$. Preliminary results of an ongoing trial suggest a survival benefit of low dose dexamethasone in patients requiring at least oxygen treatment ${ }^{24}$. Ongoing clinical trials of corticosteroids in COVID-19 might provide further insights.

\section{Plasma exchange and IVIg}

Plasma exchange or IVIg are used for acute therapy in many neuroimmunological diseases. In some diseases, such as chronic inflammatory demyelinating polyneuropathy (CIDP) and myasthenia gravis, these therapies are used repeatedly with or without other DMTs.

Plasma exchange usually requires the patient to attend hospital, sometimes requires a central venous catheter with an associated risk of infection and reduces levels of the patient's own immunoglobulins and complement factors for several weeks, which could affect COVID-19 response and outcome. IVIg usually requires the patient to attend hospital or an infusion unit, although home administration is possible in some cases (depending also on the regulatory environment). This treatment does
Box 1 | Development of a Delphi-like process

The homepages of national and international neurological societies and patient organizations were screened for relevant recommendations, statements and agreements (Supplementary Table 1). The formats and content of the search results were heterogeneous, ranging from short, general advice to very detailed disease-specific and, in some instances, product-specific recommendations.

On the basis of the frequency with which topics were addressed in the relevant literature - probably a reflection of the most urgent real-world needs - key questions were developed. Answers to these questions were drawn out of the literature and an approval rate was calculated (see the figure). A summary of each question, the relevant answer/recommendation and level of consensus are presented in TABLE 1.

Web-based search for available recommendations, publications and statements
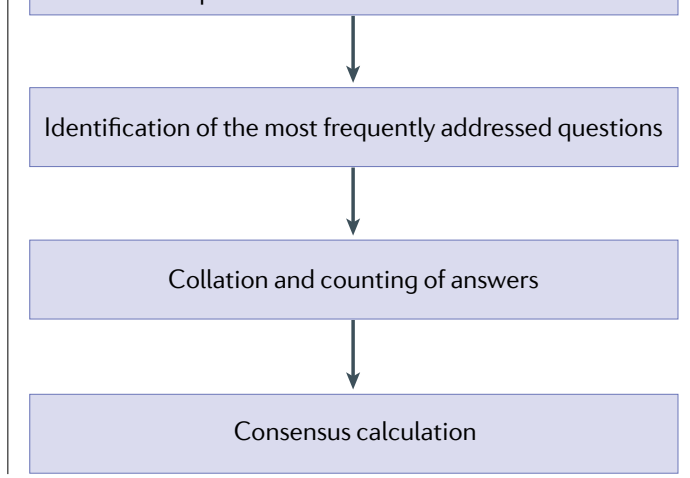

not lower immunoglobulin levels and arguably has no compromising effects related to COVID-19.

Discussion in the literature about the use of plasma exchange and IVIg in the context of COVID-19 is limited, but overall, the risk of increasing the likelihood of infection or of severe disease is considered mild (TABLE 1, Question 14) and is likely to offset risks associated with the neuroimmunological disease. Use of plasma exchange or IVIg can reduce the need for concurrent corticosteroid use and accelerate recovery and discharge from hospital, thereby reducing time in hospital and the related risks. Subcutaneous immunoglobulin can be used for CIDP and enables home administration that avoids the risk of additional exposure to SARS-CoV-2.

Beneficial effects of IVIg in COVID-19 are highly unlikely if standard, SARS-CoV-2-naive immunoglobulin preparations are used, although the immunomodulatory effects and a consequent reduction in antibody-dependent hyperinflammation have the potential to be beneficial ${ }^{25,26}$. By contrast, some evidence suggests that IVIg might increase the risk of thrombosis and substantial thrombotic complications, including multifocal stroke, in COVID-19 (REF. $\left.{ }^{27}\right)$.

\section{Serious exacerbations}

In neuromyelitis optica spectrum disorders (NMOSD), disability is generally relapse-related ${ }^{28}$ and early treatment of relapses improves outcomes ${ }^{29}$. Consequently, any risk of COVID-19 that is associated with corticosteroid pulse therapy or plasma exchange is likely to 
Table 1 | Consensus on management of neuroimmunological disease during the COVID-19 pandemic

\begin{tabular}{|c|c|}
\hline $\begin{array}{l}\text { Question } \\
\text { no. }\end{array}$ & Question \\
\hline \multicolumn{2}{|c|}{ More than ten recommendations, consensus $\geq 90 \%$} \\
\hline 1 & $\begin{array}{l}\text { Should patients with neuroimmunological } \\
\text { disease follow local recommendations? }\end{array}$ \\
\hline 2 & $\begin{array}{l}\text { Should patients with functionally relevant } \\
\text { disease deterioration be treated with } \\
\text { corticosteroids? }\end{array}$ \\
\hline 3 & $\begin{array}{l}\text { Should patients without SARS-CoV-2 infection } \\
\text { stop immune therapy? }\end{array}$ \\
\hline
\end{tabular}

$4 \quad$ Are patients who are receiving IFN $\beta$ at increased risk of SARS-CoV-2 infection?

$5 \quad$ Are patients who are receiving glatiramer acetate at increased risk of SARS-CoV-2 infection?

6 Are patients who are receiving fingolimod or siponimod at increased risk of SARS-CoV-2 infection?

$7 \quad$ Are patients who are receiving B cell-depleting therapies at increased risk of SARS-CoV-2 infection?

8 Are patients who are receiving alemtuzumab at increased risk of SARS-CoV-2 infection?

9 Are patients who are receiving cladribine at increased risk of SARS-CoV-2 infection?

10 Should patients start a DMT during the COVID-19 pandemic?

11 Should patients start an intensive (highly effective) therapy during the COVID-19 pandemic?

12 Should the next cycle of a therapy with a long treatment interval be delayed during the COVID-19 pandemic?

Should the next cycle of a B cell-depleting therapy be delayed during the COVID-19 pandemic?
Patients with neuroimmunological disease should follow the general local recommendations of social distancing, regular hand washing and/or disinfection, and avoiding public transport

Patients with an acute deterioration and relevant neurological deficits should be treated with a steroid pulse if there are no indications of SARS-CoV-2 infection

Patients who are on a stable immunotherapy and who do not have signs of SARS-CoV-2 infection should not stop treatment because of the COVID-19 pandemic

Treatment with IFN $\beta$ should not increase the risk of SARS-CoV-2 infection

Treatment with glatiramer acetate should not increase the risk of SARS-CoV-2 infection

Treatment with fingolimod or siponimod might increase the risk of SARS-CoV-2 infection

Treatment with B cell-depleting therapies might increase the risk of SARS-CoV-2 infection

Treatment with alemtuzumab might increase the risk of SARS-CoV-2 infection

Treatment with cladribine might increase the risk of SARS-CoV-2 infection.

Treatment initiation is possible; the decision to initiate a DMT in patients with newly diagnosed mild to moderate MS should be based on a discussion that balances disease-related, therapy-related and COVID-19 pandemic-related factors

Treatment initiation is possible; the decision to initiate a DMT in patients with newly diagnosed (highly) active MS should be based on a discussion that balances disease-related, therapy-related and COVID-19 pandemic-related factors

Delay of the next cycle of a therapy with a long treatment interval should be considered

Delay of the next cycle of a B cell-depleting therapy should be considered

$20 / 20(100 \%)$

$12 / 12(100 \%)$

$19 / 20(95 \%)$

$13 / 13(100 \%)$

$14 / 14(100 \%)$

$14 / 14(100 \%)$

$15 / 15(100 \%)$

$11 / 12(92 \%)$

\section{Fewer than ten recommendations, consensus $\geq 90 \%$}

14

Should patients with functionally relevant disease deterioration be treated with plasma exchange or IVIg?

15 Are patients who are receiving mitoxantrone at increased risk of SARS-CoV-2 infection?

16 Are patients who have undergone HSCT at increased risk of SARS-CoV-2 infection?

17 Should patients with MS have vaccinations during the COVID-19 pandemic?
Patients who require plasma exchange or IVIg for severe disease

$3 / 3(100 \%)$ deterioration should be treated - the risk of SARS-CoV-2 infection is only slightly increased

Mitoxantrone might increase the risk of SARS-CoV-2 infection

Patients who have undergone HSCT within the past 6-12 months have

$4 / 4(100 \%)$ an increased risk of SARS-CoV-2 infection

Patients with MS should have vaccinations against influenza and pneumococcus during the COVID-19 pandemic

$2 / 2(100 \%)$

Ten or more recommendations, consensus $\geq 70 \%$ but $<90 \%$

18 Are patients who are receiving natalizumab

Natalizumab should not increase the risk of SARS-CoV-2 infection

$10 / 13(77 \%)$ at increased risk of SARS-CoV-2 infection?

Fewer than ten recommendations, consensus $\geq 70 \%$ but $<90 \%$

19 Are patients with neuroimmunological disease at increased risk of SARS-CoV-2 infection?
Patients who have neuroimmunological diseases without respiratory symptoms, severe disability or dysphagia should not be at increased risk of SARS-CoV-2 infection besides any effects of immunosuppressive therapy 
Table 1 (cont.) | Consensus on management of neuroimmunological disease during the COVID-19 pandemic

\begin{tabular}{|c|c|c|c|}
\hline $\begin{array}{l}\text { Question } \\
\text { no. }\end{array}$ & Question & Answer & Consensus $^{\mathrm{a}}$ \\
\hline \multicolumn{4}{|c|}{ Ten or more recommendations addressing the topic, consensus $<70 \%$} \\
\hline 20 & $\begin{array}{l}\text { Are patients who are receiving teriflunomide } \\
\text { at increased risk of SARS-CoV-2 infection? }\end{array}$ & $\begin{array}{l}\text { Treatment with teriflunomide should not increase the risk of } \\
\text { SARS-CoV-2 infection }\end{array}$ & $8 / 12(67 \%)$ \\
\hline 21 & $\begin{array}{l}\text { Are patients who are receiving dimethyl } \\
\text { fumarate at increased risk of SARS-CoV-2 } \\
\text { infection? }\end{array}$ & $\begin{array}{l}\text { Treatment with dimethyl fumarate should not increase the risk } \\
\text { of SARS-CoV- } 2 \text { infection }\end{array}$ & $7 / 12(58 \%)$ \\
\hline 22 & $\begin{array}{l}\text { Should patients with mild or asymptomatic } \\
\text { COVID-19 or those who are at high risk of } \\
\text { exposure stop immunotherapy? }\end{array}$ & $\begin{array}{l}\text { Patients with mild or asymptomatic COVID-19 or who are at high risk } \\
\text { of exposure should stop immunotherapy }\end{array}$ & $5 / 12(42 \%)$ \\
\hline
\end{tabular}

be outweighed by the risk of long-term disability if not treated $^{30}$. In myasthenia gravis, acute deterioration can require plasma exchange or $\operatorname{IVIg}^{31}$ and the first reports on myasthenic crisis as a complication of COVID-19 and on the course of COVID-19 in patients with myasthenia gravis are heterogeneous ${ }^{32,33}$. Dysphagia or respiratory insufficiency can be life threatening, so IVIg or plasma exchange should be administered to patients with these symptoms ${ }^{34}$. Results are currently collected in various registries, with increasing numbers providing a better repository for evaluations; these registries include: Coronavirus and MS reporting database (COVIMS), Lean European Open Survey on SARS-CoV-2 Infected Patients (LEOSS) and COVID-19 Associated Risks and Effects in Myasthenia Gravis (CARE-MG).

\section{General chronic immunosuppression}

Approved therapies are available for few neuroimmunological diseases other than MS, so most are likely to be treated with general immunosuppressive medications, such as corticosteroids, azathioprine, mycophenolate, methotrexate and rituximab, with adjunctive IVIg and plasma exchange.

Exceptions include neurosarcoidosis, giant cell arteritis (GCA) and NMOSD, for which infliximab, tocilizumab and eculizumab, respectively, can be used. In our opinion, these drugs should not substantially increase the risk of serious COVID-19, but data from registries are needed. Similarly, life-threatening conditions such as vasculitis and autoimmune encephalitis can necessitate use of potent immunosuppression with cyclophosphamide, sometimes in combination with rituximab and other drugs. In these conditions, the severity and potentially irreversible nature of the underlying diseases should take priority over probable increases in the risk of severe COVID-19.

Long-term oral corticosteroid treatment for several diseases, including rheumatoid arthritis, systemic lupus erythematosus (SLE) and GCA, clearly increases the risk of serious infections for which the patient requires hospitalization, although data in most neuroimmunological diseases are less cohesive ${ }^{35-37}$. Higher doses, longer durations of therapy and older age are all additional risk factors for infection during chronic treatment with corticosteroids ${ }^{20}$. In a study of patients with immune-mediated inflammatory diseases and COVID-19 in New York, USA, patients treated with chronic corticosteroids or any small-molecule drug were more likely to require hospitalization for COVID-19 than patients who were not receiving corticosteroids (see the supplementary files in the original study) ${ }^{16}$, even though the rate of hospitalization among patients with immune-mediated inflammatory disease was not higher than among the general population. In patients with newly diagnosed CIDP, IVIg or plasma exchange are suggested in preference to corticosteroids ${ }^{38}$.

The immunosuppressive drugs azathioprine, mycophenolate and methotrexate are clearly associated with occasional atypical infections, such as progressive multifocal leukoencephalopathy (PML) and Pneumocystis jirovecii pneumonia ${ }^{39}$. These infections can occur with these drugs when used alone but are more common when the drugs are used as combined immunotherapies, such as after organ transplantation. These drugs are also associated with a small increase in the relative risk of all serious infections in conditions such as SLE, but the risk is smaller than that with corticosteroids ${ }^{35}$. Whether azathioprine, mycophenolate and methotrexate should be stopped or their dose reduced in the event of an active SARS-COV-2 infection or a high-risk exposure is disputed not only in the recommendations made so far (TABLE 1, Question 22) but even in the approved product information documents ${ }^{40,41}$. Authors of opinion pieces have recommended that treatment decisions with these drugs in the context of COVID-19 are decided on the basis of the immunological disease, latency of response to specific immune therapies, other patient risks and the strength of evidence that treatment will be beneficial ${ }^{26,38}$.

Long-term corticosteroid treatment should not be stopped abruptly and weaning should be done under physician supervision. In our opinion, minimizing the dosage of chronic corticosteroids is a reasonable way to reduce the risk of infection without compromising immune competence. The available evidence supports steroid-sparing immunotherapy in general practice, meaning that the introduction of an immunosuppressive agent such as mycophenolate should not be delayed if the alternative is continuation of high-dose corticosteroids, even in the context of COVID-19. 


\section{Box 2 | Factors in treatment decisions for neuroimmunological diseases}

First dimension - general factors

- Patient-related factors (including age, comorbidities, gender, personal perspective)

- Disease-related factors (including diagnosis, prognosis, disease activity, reversibility)

- Physician-related factors (including experience, personal evaluation)

- Treatment-associated factors (including immunosuppressive effects, cell depletion, reversibility)

Second dimension - phase of treatment

- Acute deterioration (corticosteroids, plasma exchange or intravenous immunoglobulin)

- Treatment initiation (time point of start, mode of action)

- Treatment continuation (dosage, possibility of delaying, safety controls, cessation)

- Treatment optimization (dosage, time point, mode of action, reversibility)

Third dimension - SARS-CoV-2-associated factors

- No infection

- Acute mild infection

- Acute severe infection

- Recovered from infection or COVID-19

- Local infection rate

- Local strategy for pandemic management

COVID-19, coronavirus disease 2019; SARS-CoV-2, severe acute respiratory syndrome coronavirus 2. with mild-to-moderate MS disease activity during the COVID-19 pandemic (TABLE 1, Questions 4 and 15). In general, interferons impair viral replication ${ }^{43}$ and previous evidence suggests that IFN $\beta$ is mildly effective in animal models of MERS when used in combination with lopinavir and ritonavir ${ }^{44}$, so the potential for IFN $\beta$ in the treatment of COVID-19 could be worth investigating.

\section{Glatiramer acetate}

Glatiramer acetate is a synthetic amino acid copolymer that contains the key amino acids from myelin basic protein ${ }^{45}$. Its mechanism of action includes attenuation and rebalancing of $\mathrm{T}$ cell responses (a shift towards the type $2 \mathrm{~T}$ helper cell phenotype), cytokine secretion and B cell function ${ }^{46,47}$. Glatiramer acetate is not immunosuppressive and is not associated with increased risk of infection. The consensus in the literature is that treatment with glatiramer acetate should not increase the risk of severe COVID-19 in patients with MS (TABLE 1, Question 4 and 10), so is another good option for initiation of DMT in patients with mild-to-moderate disease during the COVID-19 pandemic.

\section{Teriflunomide}

Teriflunomide selectively and reversibly inhibits dihydroorotate dehydrogenase (DHODH), a key mitochondrial enzyme in the de novo pyrimidine synthesis pathway ${ }^{48}$. By influencing mitochondrial metabolism, teriflunomide causes selective immune suppression by inhibiting growth and division of rapidly dividing cells, including activated $\mathrm{T}$ cells and $\mathrm{B}$ cells ${ }^{49}$. Owing to the mechanism of action, mild decreases in lymphocyte counts have been observed during teriflunomide treatment but rates of grade 2 lymphopenia and serious infections are low, even after long-term therapy ${ }^{50}$.

Expert opinions on whether teriflunomide can increase the risk of severe COVID-19 are heterogeneous, probably owing to the known risk of lymphopenia. Decreases in lymphocyte counts occur within 6 months of teriflunomide initiation but are mostly mild when the dosing scheme approved for MS is used. On this basis, most, but not all, recommendations suggest that teriflunomide should not increase the risk of severe COVID-19 (TABLE 1, Questions 20 and 10). Importantly, teriflunomide has a long half-life ( $\sim 16$ days in the plasma) and elimination takes time because the drug persists in the enterohepatic cycle, so health-care providers and patients should keep these factors in mind if stopping teriflunomide is considered upon SARS-CoV-2 infection.

Teriflunomide can inhibit replication of EpsteinBarr virus, cytomegalovirus (CMV), herpes simplex virus (HSV) 1 and 2, and poliovirus ${ }^{51-54}$. These observations suggest that $\mathrm{DHODH}$ inhibition could be a therapeutic strategy for COVID-19 (REF. ${ }^{55}$ ).

a and IFN $\beta 1 b$ are recombinant cytokines increase the cytotoxicity of natural killer cells, the phagocytic activity of macrophages and the antibodydependent cytotoxicity of leukocytes ${ }^{42}$. National and international neurological societies agree that treatment with IFN $\beta$ does not increase the risk for patients during the COVID-19 pandemic. Interferons are not considered to be immunosuppressive and could therefore be a safe option for initiation of DMT in patients

\section{Dimethyl fumarate}

Dimethyl fumarate reduces the absolute lymphocyte count, although the mechanisms of action are not fully understood. Memory CD8 ${ }^{+} \mathrm{T}$ cells are most profoundly affected but levels of pro-inflammatory $\mathrm{CD} 4^{+} \mathrm{T}$ cell subsets, and B cell subsets are also decreased, creating a bias 
towards an anti-inflammatory state ${ }^{56,57}$. Lymphocytes normally decrease within the first 6 months of treatment but moderate or severe prolonged lymphopenia has been observed in $2-9 \%$ of patients ${ }^{58}$. Lymphopenia is considered to be a risk factor for dimethyl fumarate-associated PML (caused by JC virus) ${ }^{59}$, so dimethyl fumarate therapy must be stopped if lymphocyte counts are $\leq 500$ cells/ $\mu$ l. In addition, in rare cases, low lymphocyte counts in patients receiving dimethyl fumarate have been associated with HSV encephalitis ${ }^{60}$. Though the risk of lymphopenia after initiation of dimethyl fumarate treatment is a potential risk for COVID-19, most national and international recommendations suggest that dimethyl fumarate treatment should not increase the risk of severe COVID-19 (TABLE 1, Question 21).

\section{Natalizumab}

Natalizumab was the first monoclonal antibody to be approved for the treatment of relapsing-remitting MS. The target of the antibody is integrin $\alpha 4$, which mediates immune cell adhesion and migration via interactions with vascular cell adhesion molecule 1 and fibronectin ${ }^{61}$. In general, infection rates among patients receiving natalizumab are low ${ }^{10,62}$ besides a slight increase in the risk of HSV infection. The initial trial of natalizumab did suggest an increased risk of pneumonia ${ }^{63}$ but this observation does not seem to have been replicated in real-world series ${ }^{62,64}$. The most concerning adverse effect of natalizumab is a low risk of PML, which is influenced by the JC virus antibody index, the duration of therapy and prior exposure to immune-suppressive agents ${ }^{65}$.

Most, but not all, international and national recommendations suggest that natalizumab therapy should not increase the risk of severe COVID-19 (TABLE 1, Question 18). On this basis natalizumab is a candidate for patients who need to start on a high-efficacy therapy during the COVID-19 pandemic, and natalizumab should be continued for all patients who are already

\section{Box $3 \mid$ Summary of the main results of the Delphi-like process}

\section{Aspects for which consensus is strong}

- Patients without infection should not stop ongoing immunotherapy.

- In acute deterioration of neuroimmunological conditions, corticosteroids, intravenous immunoglobulin or plasma exchange can be administered.

- In MS, glatiramer acetate and IFN $\beta$ do not have a negative effect in relation to SARS-CoV-2 infection or the course of COVID-19.

- In active MS, natalizumab is considered to be associated with a lower risk of COVID-19 than other high-efficacy disease-modifying therapies.

- In MS, sphingosine 1-phosphate receptor modulators and cell-depleting therapies increase the risk of SARS-CoV-2 infection.

- In MS, postponement of cell-depleting therapy can be considered in patients continuing on therapy.

\section{Aspects for which consensus is low}

- Whether teriflunomide and dimethyl fumarate increase the risk of SARS-CoV-2 infection

- Whether patients with mild or asymptomatic COVID-19 and those with a high risk of exposure to SARS-CoV-2 should stop immunotherapy during the COVID-19 pandemic

- How disease-modifying therapies influence the course of severe COVID-19 in which overwhelming inflammation occurs

COVID-19, coronavirus disease 2019; MS, multiple sclerosis; SARS-CoV-2, severe acute respiratory syndrome coronavirus 2 . receiving the therapy (TABLE 1, Question 11). Indeed, cessation or interruption of natalizumab treatment carries a considerable risk of MS disease exacerbation or rebound activity in patients with highly active $\mathrm{MS}^{66}$, the consequences of which are likely to outweigh the risk of COVID-19. Natalizumab has been suggested as a bridging therapy (a drug that can be used for an immediate effect owing to its fast onset of activity and high efficacy but that is not necessarily considered a long-term option) during the COVID-19 pandemic, and the first reports of SARS-CoV-2 infection in patients receiving natalizumab therapy have suggested no increase in risk ${ }^{67,68}$. Furthermore, extended dosing intervals can be used to limit infusions and, consequently, limit the exposure of patients to hospitals ${ }^{69}$. Extended dosing intervals could also decrease the risk of COVID-19-related encephalitis by allowing some CNS immunosurveillance.

The CNS is not thought to be the primary site or a major site of SARS-CoV-2 infection, although CNS manifestations, including dizziness, headache, impaired consciousness, acute cerebrovascular disease, ataxia and seizures, have been reported in patients with severe COVID-19 (REFS ${ }^{70,71}$ ). Some evidence indicates that integrins have a role in host cell entry by SARS-CoV-2 $\left(\mathrm{REFS}^{72,73}\right)$, suggesting that natalizumab could even have beneficial effects in cases where CNS infection does occur.

\section{Fingolimod, siponimod and ozanimod}

Fingolimod, siponimod and ozanimod are sphingosine 1-phosphate receptor (S1PR) modulators that result in downregulation of S1PR, which prevents egress of lymphocytes from lymphoid tissue. All three drugs are known to decrease blood lymphocyte counts, but importantly this effect does not reflect the death or loss of lymphocytes, especially not those in the bone marrow - it means that these cells might still be recruitable and functional but entrapped. Fingolimod treatment has been associated with increased rates of varicella zoster virus (VZV) activation or reactivation ${ }^{74}$, and other viral infections, such as PML, have been described in rare cases $^{75-77}$. Siponimod and ozanimod are more selective for the S1PR than is fingolimod but they have similar effects on lymphocyte counts and have been associated with similar infections ${ }^{78-81}$. The effects of these drugs are reversible and their half-lives are short but the effects of S1PR modulators on lymphocyte count can persist for several weeks to months ${ }^{82}$.

National and international experts foresee a mild-tomoderate increase in the risk of SARS-CoV-2 infection among patients who are treated with fingolimod, siponimod or ozanimod (TABLE 1, Question 6). However, cessation or interruption of therapy with S1PR modulators can trigger a severe disease rebound with a high risk of relapse, so patients who are already receiving fingolimod, siponimod or ozanimod should continue their therapy. For patients who need to switch therapy or start a new therapy, other treatment options should be considered.

Fingolimod also has the potential to be of benefit in COVID-19 on the basis that secondary invasion of alveoli by lymphocytes is thought to be harmful in this disease ${ }^{83}$ and could be prevented by fingolimod. 
A clinical trial of this approach is currently recruiting patients $^{84}$. The effect of S1PR modulation on the outcome of the second phase of COVID-19 is the question under investigation.

\section{Immune-depleting and repopulating therapies}

$B$ cell-depleting therapies. Ocrelizumab, ofatumumab and rituximab are monoclonal CD20 antibodies that deplete $\mathrm{CD} 20^{+} \mathrm{B}$ cells and small populations of $\mathrm{CD} 20^{+}$ $\mathrm{T}$ cells. Inebilizumab is a CD19 antibody that depletes $\mathrm{B}$ cells expressing CD19. Ocrelizumab is approved for the treatment of relapsing-remitting and primary progressive MS, ofatumumab is expected to be approved soon, and rituximab is used off-label in several neuroimmunological diseases, including NMOSD, myasthenia gravis, immune neuropathies and MS. Inebilizumab has recently been approved for the treatment of NMOSD.

$B$ cell depletion reduces humoral and cellular immunity. Immunoglobulin levels can decrease as the duration of treatment increases, but broad cytopenia rarely occurs $^{85}$. In phase III studies, ocrelizumab treatment was associated with a slight increase in the risk of infections (generally mild to moderate and none unusual, such as opportunistic infections $)^{86}$. Ofatumumab was not associated with an increased risk of infections in the phase II study programme, but longer term observations are currently lacking ${ }^{87}$. Similarly, although serious infections were not associated with rituximab treatment in phase III studies, real-world use has revealed some risks - long-term use of rituximab is associated with an increased risk of severe infection, and rituximab and ocrelizumab have been associated with reactivation of hepatitis $\mathrm{B}$ and $\mathrm{C}$ viruse ${ }^{10,88,89}$. In addition, PML has been reported in several patients receiving rituximab for various diseases and PML was associated with ocrelizumab in one patient (as of April 2020), although multiple influencing factors have to be considered in this case $\mathrm{e}^{90}$.

The first reports on the impact of B cell-depleting therapy on COVID-19 have been published ${ }^{14,67,91}$. In 232 patients with MS and suspected or proven COVID-19, the severity of COVID-19 was classified as mild (no or mild pneumonia) in 222 (96\%), severe (shortness of breath, respiratory rate $\geq 30$ breaths/min, blood oxygen saturation $\leq 93 \%, \mathrm{PaO}_{2}: \mathrm{FiO}_{2}<300 \mathrm{mmHg} / \%$, and an increase in lung infiltrates of $>50 \%$ within $24-48 \mathrm{~h}$ ) in $4(2 \%)$ and critical (respiratory failure, septic shock and multiple organ dysfunction or failure) in $6(3 \%)$. Of the 6 patients with critical illness, 1 recovered and 5 died. Of the 28 patients receiving a B cell-depleting therapy, $3(10 \%)$ developed a severe or critical disease course ${ }^{14}$. In addition, 100 patients with confirmed or suspected SARS-CoV-2 infection have been studied within pharmacovigilance reporting conditions by Roche ${ }^{92}$; 26 of these patients were admitted to hospital and 13 remained in hospital at the time of writing. Five patients were classified as critically ill and needed intensive care. Among the limited data currently available, outcomes of COVID-19 during B cell-depleting therapy range from mild disease to death but - although the number of patients is low - rates of critical illness and death do not seem to be increased dramatically relative to the wider population $^{93}$. This observation could be explained by the fact that, although B cell-depleting therapies are thought to increase susceptibility to acute respiratory infections ${ }^{94}$, antibody-mediated inflammation and a cytokine storm are thought to mediate severe COVID-19 outcomes, so $B$ cell depletion might not necessarily be associated with more severe disease $\mathrm{e}^{95}$.

International and national recommendations suggest that B cell-depleting therapy is likely to increase the risk of severe COVID-19 (TABLE 1, Question 12) but that the risk level depends on the duration of therapy and the time since the last dose because immunoglobulin deficiency occurs more frequently after 2-3 years of treatment and circulating drug levels are likely to decline during the treatment interval. Depletion of B cells in the peripheral circulation lasts for at least 5-6 months after the last dose and full recovery of B cells after a course of treatment with ocrelizumab takes up to 72 weeks.

On the basis of current knowledge, postponement of the next dose of B cell-depleting therapy is advisable for some patients with MS (TABLE 1, Question 13), especially those with additional risk factors and progressive disease, or unsafe infusion environments. However, the pandemic is ongoing and therapy cannot be delayed indefinitely - waiting until disease activity returns is not advisable. Levels of peripheral B cells and the recovery of these levels is one possible indicator that can be monitored to assess the urgency of the next infusion. In principle, CD20 antibody therapy could be initiated during the COVID-19 pandemic in suitable patients (for example, those with high disease activity, no comorbidities and a younger age), but the risk to benefit ratio should be taken into account, especially in older patients and those with comorbidities. B cell depletion in older patients with progressive MS and in patients with mild MS and no activity should be viewed with particular caution because these patients are least likely to benefit from anti-CD20 therapies.

Another issue that must be considered with B celldepleting therapy is the nature of the immune response to either a primary infection with SARS-CoV-2 or a primary vaccination, should a vaccine be developed. Rituximab treatment is known to inhibit the primary antibody response to a neoantigen to a greater extent than the secondary response to a known antigen ${ }^{96}$, whereas in one patient undergoing ocrelizumab therapy, $\mathrm{B}$ cell and $\mathrm{T}$ cell responses to primary VZV infection were not impaired ${ }^{97}$. In 68 patients receiving ocrelizumab, pre-existing humoral immunity was not affected by $\mathrm{B}$ cell depletion and even if the humoral response to a neoantigen or vaccine was attenuated, patients were able to mount humoral responses ${ }^{98}$. However, the primary immune response and the associated class shifting of immunoglobulins to IgG and IgA might be affected more than the response to a secondary immunization (contact with the antigen for a second time) ${ }^{98}$, which could result in a cohort of patients who have little immunity to SARS-CoV-2 infection despite vaccination.

Alemtuzumab. Alemtuzumab is a monoclonal antibody to CD52 that leads to depletion of T cells and B cells ${ }^{99,100}$. Treatment impairs cellular and humoral immunity; 
the extent of impairment depends on the time since the last dose and the number of treatment cycles $^{101,102}$. Alemtuzumab changes the immune system from a proinflammatory state to a regulatory state, and the effect is long-lasting; low lymphocyte counts - specifically low $\mathrm{CD}^{+} \mathrm{T}$ cell counts - can last for months and neutropenia occurs in some patients ${ }^{103}$.

In the first few months after dosing, the risk of HSV re-activation is increased ${ }^{104}$. Frequent CMV infection and VZV activation have also been reported and immunity against intracellular bacteria (for example, listeria) seems to be compromised during and shortly after infusion periods ${ }^{104}$. Analysis of long-term data has shown that the incidence of infections decreases substantially 2 years after the last dose of alemtuzumab and that most infections after this time were mild ${ }^{104}$. Secondary autoimmunity after CD52 depletion can occur up to 4 years after the last infusion cycle but long-term extension studies do not indicate altered immune competence or high infection rates.

The consensus among national and international recommendations is that patients receiving alemtuzumab have an increased risk of severe COVID-19 (moderate to high confidence) owing to the profound effects of the drug on the immune system (TABLE 1, Question 8). The absolute risk of infection clearly depends on the time since the last dose - the highest risk is in the weeks that follow immune depletion ${ }^{102}$. Therefore, alemtuzumab should only be used with caution in the COVID-19 pandemic; careful evaluation of the risks and benefits in a particular patient is needed.

Cladribine tablets. Cladribine is a synthetic purine nucleoside analogue that inhibits DNA synthesis selectively, mainly in circulating $\mathrm{T}$ cells and $\mathrm{B}$ cells. This action leads to an extended decrease in lymphocyte counts (depletion) with minimal effect on innate immunity, followed by gradual repopulation of the lymphocytes ${ }^{105,106}$. Depletion of CD $19^{+} \mathrm{B}$ cells occurs earlier and to a greater extent than that of $\mathrm{CD}^{+}$and $\mathrm{CD}^{+} \mathrm{T}$ cells ${ }^{107,108}$. However, depletion is less profound than that seen with alemtuzumab $^{107,109-111}$, which might account for the fact that the rates and severity of infection are generally lower with cladribine and usually occur within the first few months after dosing. Cladribine might affect immune competence against intracellular bacteria (for example, Mycobacterium tuberculosis) ${ }^{112}$.

No data are yet available on the impact of cladribine tablets on COVID-19 but consensus among national and international societies is that cladribine can increase the risk of severe COVID-19 (moderate to high confidence), depending on the time since the last dose - a shorter time since the last dose is associated with a higher risk (TABLE 1, Question 9). Given that cladribine has long-lasting effects and the interval between treatment cycles is 1 year, a second cycle of treatment can be delayed if the patient is stable, but this option depends on the local infection rate (TABLE 1, Question 12) - if the local infection rate is high, delay should be considered. Cladribine tablets can be initiated in suitable patients (for example, patients with high disease activity, younger age and no comorbidities) during the COVID-19 pandemic, but the risk-to-benefit ratio should be considered, especially for older patients and those with comorbidities.

Haematopoietic stem cell therapy. Haematopoietic stem cell therapy (HSCT) is an off-label treatment used for $\mathrm{MS}^{113}$ and, in rare cases, other neuroimmunological conditions ${ }^{100,114}$. Preparation for the transplantation involves profound immunosuppression or immunoablation, which carries a high risk of infection. The consensus is that patients who underwent HSCT within the past 6-12 months are at risk of severe COVID-19 (TABLE 1, Question 16).

\section{Asymptomatic, mild and severe COVID-19 Asymptomatic or mild COVID-19}

Worldwide SARS-CoV-2 infection rates are increasing, and evidence indicates that a high number of people who are infected have no symptoms ${ }^{115}$. For example, in a study conducted in Iceland, $43 \%$ of people who tested positive for SARS-CoV-2 exhibited no symptoms ${ }^{116}$. On this basis, there are undoubtedly patients who are receiving immunotherapy and are unknowingly infected with SARS-CoV-2.

No consensus exists about how to manage ongoing immunotherapy in patients who are at high risk of exposure to SARS-CoV-2, have asymptomatic infection or who develop mild symptoms of COVID-19 (TABLE 1, Question 22). In making the decision to stop or continue immunotherapy, health-care providers should consider the duration of the therapeutic effects, the risk of relapse, the postulated duration of COVID-19 and patient-related factors (BOX 2).

In the literature, general agreement exists that local recommendations for disease control, such as social distancing, should be followed by individuals receiving immunotherapy (TABLE 1, Question 1). However, there is no clarity about regular laboratory tests for safety monitoring, which might require the patient to travel to a collection facility or hospital, thereby increasing the risk of exposure to SARS-CoV-2. The risk of infection in local infusion centres and hospitals depends strongly on local infection rates and the precautions taken; in high-risk areas, postponing treatment should be considered whenever possible. If collection and infusion are possible at home, this approach would be preferable. The risks of complying with monitoring procedures versus those of failing to do so depend heavily on local circumstances.

Another important consideration is the effect of immunotherapy on SARS-CoV-2 viral shedding. The time for which viral shedding continues can vary widely, and a high viral load can persist in otherwise healthy people without comorbidities for $>2$ months $s^{117}$, and evidence from previous studies suggests that immunosuppression can prolong viral shedding further. For example, viral shedding of MERS-CoV was prolonged in immunocompromised macaques even though the disease course was not more severe in these animals than in immunocompetent animals ${ }^{118}$. In humans, prolonged viral shedding of various respiratory viruses has been described in patients receiving immunotherapy after transplantation ${ }^{119}$. The possibility of prolonged shedding of SARS-CoV-2 could, therefore, 
influence recommended isolation periods and re-testing strategies during convalescence for people receiving immunotherapy.

Although no vaccination against SARS-CoV-2 is yet available, viral co-infection has been found in $13.1 \%$ of individuals who are positive for SARS-CoV-2 (REF. ${ }^{120}$ ) and bacterial and fungal co-infections have been observed in at least $8 \%{ }^{121}$. Therefore, vaccinations against influenza and/or pneumococcus might be helpful in patients receiving immunotherapy to reduce the risk, but few recommendations have been made on this topic (TABLE 1, Question 7). An important question for future management is whether and how the efficacy of a SARS-CoV-2 vaccine might be influenced by immunotherapy. Responses to vaccinations can be altered by immunotherapy, depending on the particular mechanism of action of the drug. For example, response rates to seasonal influenza vaccination were low among patients with MS who were being treated with natalizumab or fingolimod ${ }^{122,123}$. In patients who are receiving immune-depleting therapies, vaccination response rates are attenuated but some extent of immune response is seen even after B cell depletion ${ }^{98}$.

The possibility of altered responses to certain types of vaccination with some immunotherapies is already included in the 'checklist' for de-risking immunotherapy ${ }^{124}$. Ideally, vaccinations are performed before initiation of an immune therapy, particularly highefficacy immunotherapy. In the case of immunedepleting, pulsed therapies, vaccinations should be administered during phases of immune system recovery ( $\geq 6$ months after a course of immune-depleting therapy with alemtuzumab; $\geq 2-4$ months after a course of CD20-depleting therapy). This approach holds true for seasonal vaccines (for example influenza). To date, the potential for altered vaccination responses has not been a major factor in choosing immunotherapies, as the problem can be mitigated by vaccinating before therapy initiation or some immunotherapies still allow sufficient immune responses. Whether the same will hold true in the context of a SARS-CoV-2 vaccine remains to be determined when the properties of any vaccine are known.

\section{Severe COVID-19}

Neuroimmunological disease alone is probably not a risk factor for symptomatic infection with SARS-CoV-2 or for severe COVID-19 (TABLE 1, Question 19). However, some immunotherapies seem to increase susceptibility to SARS-CoV-2 infection, so stopping therapy might seem reasonable based on the assumption that immunotherapy and associated dampening of primary infection control (the innate immune system) could negatively impact the disease course. However, the influence of immunotherapy on the risk of SARS-CoV-2 infection might differ from that on the risk of a severe disease course.

Innate immunity, natural killer cells and type I interferons are considered to be the first line of defence in viral infection ${ }^{125}$. Adaptive immune responses to viral infections consist of humoral immunity involving antibodies and cytotoxic T cells. In severe COVID-19, however, the host response to SARS-CoV-2 infection leads to fulminant inflammation that includes peripheral lymphopenia, sequestration of mononuclear cells in the infected tissues and reduction of $\mathrm{CD}^{+}$lymphocytes in the blood ${ }^{126}$. In addition, upregulation of cytokines can lead to a cytokine storm ${ }^{127}$. In this second phase of severe COVID-19, some immunotherapies might have the potential to attenuate or even prevent critical illness ${ }^{93,128}$, although this potential is largely theoretical at present and there is a long history of similar hypotheses that have rarely come to fruition.

Low levels of immunoglobulins are associated with $\mathrm{B}$ cell-depleting therapies and anti-CD52 therapy ${ }^{101}$. These low levels are unlikely to reduce primary responses to SARS-CoV-2 infection because these responses involve $\mathrm{CD}^{+} \mathrm{T}$ cells and innate immune components. However, low levels of immunoglobulins could attenuate antibody-dependent cellular cytotoxicity, which is thought to contribute to severe secondary hyperinflammation in COVID-19. This hypothesis has been proposed as an explanation as to why higher IgG levels are a risk factor for severe COVID-19 (REF. ${ }^{129}$ ).

Individual cytokines can be blocked, with the potential to attenuate the cytokine storm in COVID-19. IL-6 receptor antagonists (for example, tocilizumab), IL-1 blockers (for example, anakinra) and Janus kinase (JAK) inhibitors (for example, baricitinib) are either being tested in clinical trials or have been proposed as therapeutic agents to target the excessive inflammation ${ }^{130,131}$. Another target of therapies that are currently being trialled in COVID-19 is the complement system, activation of which might contribute to the secondary inflammatory response and microvascular injury in patients with severe COVID-19 (REF. ${ }^{132}$ ). Reports of patients with COVID-19 being successfully treated with the compstatin-based complement inhibitor AMY-101 $\left(\right.$ REF. ${ }^{133}$ ) and the anti-C5 therapy eculizumab have been published $^{134}$.

Early reports indicate that patients who are receiving immunotherapy after solid organ transplantation or for inflammatory bowel disease do not have an increased risk of developing severe COVID-19 (REFS ${ }^{135,136}$ ). On this basis, one possibility is that an increased risk of infection due to the immunotherapy is outweighed by the anti-inflammatory effects of immunosuppression ${ }^{137}$.

\section{Factors to consider in decision making}

The COVID-19 pandemic presents a new situation that challenges the field of neuroimmunology. Owing to the rapid and novel evolution of the pandemic, decisionmaking processes remain largely non-evidence-based but are assisted by several national and international society recommendations and opinion papers. Nevertheless, several relevant questions are not addressed and conflicting advice has been given about some aspects. The decision-making process involves several dimensions and key contributing factors (BOX 2). The complexities of the existing dimensions - dimensions one and two - are now complicated further by a third dimension, which comprises SARS-Cov-2-related factors.

Importantly, however, the COVID-19 pandemic has not led to a complete modification of how the initiation, 
continuation and optimization of neurological immunotherapy should be managed. The novelty comes from what is not known about SARS-CoV-2 and from the different conditions that individual health-care systems face. Nevertheless, it is unlikely that all patients receiving immunotherapy have a substantially increased risk of severe COVID-19, except for patients who are receiving potent immunosuppression or who have confounding factors that further increase the risk of poor outcomes (such as old age, smoking or cardiovascular comorbidities) $)^{138}$.

\section{Conclusions}

During the COVID-19 pandemic, initiation, continuation and optimization of treatment for neuroimmunological disease that requires disease-modifying immunotherapy should be based on the features of the underlying disease, the characteristics of the patient, and caregiver considerations. In general, delaying treatment could risk a poor long-term prognosis and persistent disability, especially with very active neuroimmunological disease or when the pathology is irreversible.

Some questions about the use of disease-modifying immunotherapies in the context of the COVID-19 pandemic cannot currently be answered on the basis of good evidence or even on the basis of consensus, although a strong consensus exists for some questions (BOX 3). Collection of data in registries of all patients with neuroimmunological disease and SARS-CoV-2 infection should be an important goal to gain additional information on outcomes and inform future management approaches.

In addition, most of the available information in this field - demonstrated by the sources included in our Delphi-like analysis - relates to MS, leaving many unanswered questions and/or limited consensus in relation to other areas of neuroimmunology. These questions include whether people with neuroimmunological disease are at increased risk of severe COVID-19; which immunotherapies and which comorbidities and confounding factors increase the risk of severe COVID-19; and which therapies should be discontinued in those with confirmed SARS-CoV-2 infection if possible. Until we know the answers to these questions, the practice of neuroimmunology during the COVID-19 pandemic requires a thorough understanding of first principles and a careful balancing of the risks that are particular to each individual patient.

Published online 8 July 2020
1. Zhu, N. et al. A novel coronavirus from patients with pneumonia in China, 2019. N. Engl. J. Med. 382, 727-733 (2020)

2. Zhou, P. et al. A pneumonia outbreak associated with a new coronavirus of probable bat origin. Nature $\mathbf{5 7 9}$ 270-273 (2020)

3. Siordia, J. A. Epidemiology and clinical features of COVID-19: a review of current literature. J. Clin. Virol. 127, 104357 (2020).

4. Iwasaki, A. \& Yang, Y. The potential danger of suboptimal antibody responses in COVID-19. Nat. Rev. Immunol. 20, 339-341 (2020).

5. Herold, T. et al. Elevated levels of IL-6 and CRP predict the need for mechanical ventilation in COVID-19. J. Allergy Clin. Immunol. https://doi.org/10.1016/ j.jaci.2020.05.008 (2020)

6. Gorse, G. J., Donovan, M. M. \& Patel, G. B. Antibodies to coronaviruses are higher in older compared with younger adults and binding antibodies are more sensitive than neutralizing antibodies in identifying coronavirus-associated illnesses. J. Med. Virol. 92 , 512-517 (2020).

7. Wang, L., Wang, F.-S. \& Gershwin, M. E. Human autoimmune diseases: a comprehensive update. J. Intern. Med. 278, 369-395 (2015).

8. Kovarik, J. From immunosuppression to immunomodulation: current principles and future strategies. Pathobiology 80, 275-281 (2013).

9. Willis, M. D. \& Robertson, N. P. Multiple sclerosis and the risk of infection: considerations in the threat of the novel coronavirus, COVID-19/SARS-CoV-2. J. Neurol. 267, 1567-1569 (2020).

10. Luna, G. et al. Infection risks among patients with multiple sclerosis treated with fingolimod, natalizumab, rituximab, and injectable therapies. JAMA Neurol. 77, 184-191 (2020)

11. Fu, Y., Cheng, Y. \& Wu, Y. Understanding SARS-CoV-2mediated inflammatory responses: from mechanisms to potential therapeutic tools. Virol. Sin. https://doi.org/ 10.1007/s12250-020-00207-4 (2020).

12. Jamilloux, Y. et al. Should we stimulate or suppress immune responses in COVID-19? Cytokine and anticytokine interventions. Autoimmun. Rev. 19, 102567 (2020).

13. Steinberg, K. P. et al. Efficacy and safety of corticosteroids for persistent acute respiratory distress syndrome. N. Engl. J. Med. 354, 1671-1684 (2006).

14. Sormani, M. P. Italian study group on COVID-19 infection in multiple sclerosis. An Italian programme for COVID-19 infection in multiple sclerosis. Lancet Neurol. 19, 481-482 (2020).
15. Minotti, C., Tirelli, F., Barbieri, E., Giaquinto, C. \& Donà, D. How is immunosuppressive status affecting children and adults in SARS-CoV-2 infection? A systematic review. J. Infect. 81, e61-e66 (2020).

16. Haberman, R. et al. Covid-19 in immune-mediated inflammatory diseases - case series from New York. N. Engl. J. Med. https://doi.org/10.1056/ NEJMc2009567 (2020).

17. Boulkedid, R., Abdoul, H., Loustau, M., Sibony, O. \& Alberti, C. Using and reporting the Delphi method for selecting healthcare quality indicators: a systematic review. PLoS One 6, e20476 (2011).

18. Cain, D. W. \& Cidlowski, J. A. Immune regulation by glucocorticoids. Nat. Rev. Immunol. 17, 233-247 (2017).

19. Fan, P. T. et al. Effect of corticosteroids on the human immune response: comparison of one and three daily $1 \mathrm{gm}$ intravenous pulses of methylprednisolone. J. Lab. Clin. Med. 91, 625-634 (1978).

20. Youssef, J., Novosad, S. A. \& Winthrop, K. L. Infection risk and safety of corticosteroid use. Rheum. Dis. Clin. North. Am. 42, 157-176 (2016).

21. Arabi, Y. M. et al. Corticosteroid therapy for critically ill patients with Middle East respiratory syndrome. Am. J. Respir. Crit. Care Med. 197, 757-767 (2018)

22. Russell, C. D., Millar, J. E. \& Baillie, J. K. Clinical evidence does not support corticosteroid treatment for 2019-nCoV lung injury. Lancet 395, 473-475 (2020).

23. Alhazzani, W. et al. Surviving sepsis campaign guidelines on the management of critically ill adults with coronavirus disease 2019 (COVID-19). Crit. Care Med. https://doi.org/10.1097/CCM. 0000000000004363 (2020)

24. Horby, $\mathrm{P}$ et al. Effect of dexamethasone in hospitalized patients with COVID-19: preliminary report. Preprint at medRxiv https://doi.org/10.1101/2020.06.22. 20137273 (2020).

25. Nguyen, A. A. et al. Immunoglobulins in the treatment of COVID-19 infection: proceed with caution! Clin. Immunol. 216, 108459 (2020).

26. Guidon, A. C. \& Amato, A. A. COVID-19 and neuromuscular disorders. Neurology https://doi.org/ 10.1212/WNL.0000000000009566 (2020).

27. Klok, F. A. et al. Confirmation of the high cumulative incidence of thrombotic complications in critically ill ICU patients with COVID-19: an updated analysis. Thromb. Res. https://doi.org/10.1016/j.thromres. 2020.04.041 (2020)

28. Palace, J. et al. Outcome prediction models in AQP4-IgG positive neuromyelitis optica spectrum disorders. Brain 142, 1310-1323 (2019).
29. Kleiter, I. et al. Apheresis therapies for NMOSD attacks: a retrospective study of 207 therapeutic interventions. Neurol. Neuroimmunol. Neuroinflamm 5, e504 (2018)

30. Carnero Contentti, E. \& Correa, J. Immunosuppression during the COVID-19 pandemic in neuromyelitis optica spectrum disorders patients: a new challenge. Mult. Scler. Relat. Disord. 41, 102097 (2020).

31. Sanders, D. B. et al. International consensus guidance for management of myasthenia gravis: Executive summary. Neurology 87, 419-425 (2016).

32. Delly, F., Syed, M. J., Lisak, R. P. \& Zutshi, D. Myasthenic crisis in COVID-19. J. Neurol. Sci. 414 116888 (2020).

33. Anand, P. et al. COVID-19 in patients with myasthenia gravis. Muscle Nerve 19, 1 (2020).

34. International MG/COVID-19 Working Group. et al. Guidance for the management of myasthenia gravis (MG) and Lambert-Eaton myasthenic syndrome (LEMS) during the COVID-19 pandemic. J. Neurol. Sci. 412 , 116803 (2020).

35. Feldman, C. H. et al. Serious infections among adult Medicaid beneficiaries with systemic lupus erythematosus and lupus nephritis. Arthritis Rheumatol. 67, 1577-1585 (2015).

36. Wilson, J. C. et al. Serious adverse effects associated with glucocorticoid therapy in patients with giant cell arteritis (GCA): a nested case-control analysis. Semin. Arthritis Rheum. 46, 819-827 (2017).

37. Wilson, J. C. et al. Incidence and risk of glucocorticoidassociated adverse effects in patients with rheumatoid arthritis. Arthritis Care Res. 71, 498-511 (2019).

38. Rajabally, Y. A., Goedee, H. S., Attarian, S. \& Hartung, H.-P. Management challenges for chronic dysimmune neuropathies during the COVID-19 pandemic. Muscle Nerve 62, 34-40 (2020).

39. Schmedt, N., Andersohn, F \& Garbe, E. Signals of progressive multifocal leukoencephalopathy for immunosuppressants: a disproportionality analysis of spontaneous reports within the US Adverse Event Reporting System (AERS). Pharmacoepidemiol. Drug. Saf. 21, 1216-1220 (2012).

40. Sebela Ireland Ltd. IMURAN (azathioprine): Product information. https://www.accessdata.fda.gov/ drugsatfda_docs/label/2018/016324s039lbl.pdf (2018).

41. Aspen. Australian Product Information: Azathioprine (Imuran) https://www.ebs.tga.gov.au/ebs/picmi/ picmirepository.nsf/pdf? OpenAgent\&id=CP-2010PI-06832-3 (2019)

42. Goodkin, D. E. Interferon beta-1b. Lancet 344 1057-1060 (1994). 
43. Mark, D. F., Lu, S. D., Creasey, A. A., Yamamoto, R. $\Sigma$ Lin, L. S. Site-specific mutagenesis of the human fibroblast interferon gene. Proc. Natl Acad. Sci. USA 81, 5662-5666 (1984)

44. Sheahan, T. P. et al. Comparative therapeutic efficacy of remdesivir and combination lopinavir, ritonavir, and interferon beta against MERS-CoV. Nat. Commun. 11, 222 (2020).

45. Weinstock-Guttman, B., Nair, K. V., Glajch, J. L., Ganguly, T. C. \& Kantor, D. Two decades of glatiramer acetate: from initial discovery to the current development of generics. J. Neurol. Sci. 376, 255-259 (2017).

46. Häusler, D. et al. Glatiramer acetate immune modulates B-cell antigen presentation in treatment of MS. Neurol. Neuroimmunol. Neuroinflamm 7, e698 (2020).

47. Ziemssen, T. \& Schrempf, W. Glatiramer acetate: mechanisms of action in multiple sclerosis. Int. Rev. Neurobiol. 79, 537-570 (2007).

48. Bar-Or, A. Teriflunomide (Aubagio ${ }^{\circledR}$ ) for the treatment of multiple sclerosis. Exp. Neurol. 262, 57-65 (2014).

49. Klotz, L. et al. Teriflunomide treatment for multiple sclerosis modulates $\mathrm{T}$ cell mitochondrial respiration with affinity-dependent effects. Sci. Transl. Med. 11, eaao5563 (2019)

50. Comi, G. et al. Characterizing lymphocyte counts and infection rates with long-term teriflunomide treatment: pooled analysis of clinical trials. Mult. Scler. https://doi.org/10.1177/1352458519851981 (2019)

51. Bilger, A. et al. Leflunomide/teriflunomide inhibit Epstein-Barr virus (EBV)-induced lymphoproliferative disease and lytic viral replication. Oncotarget 8 44266-44280 (2017).

52. Chon, W. J. et al. Use of leflunomide in renal transplant recipients with ganciclovir-resistant/refractory cytomegalovirus infection: a case series from the University of Chicago. Case Rep. Nephrol. Dial. 5 96-105 (2015)

53. Henao-Martínez, A. F., Weinberg, A., Waldman, W. J. $\&$ Levi, M. E. Successful treatment of acyclovir-resistant herpes simplex virus type 2 proctitis with leflunomide in an HIV-infected man. J. Clin. Virol. 54, 276-278 (2012).

54. Lamarche, C. et al. BK polyomavirus and the transplanted kidney: immunopathology and therapeutic approaches. Transplantation 100, 2276-2287 (2016).

55. Xiong, R. et al. Novel and potent inhibitors targeting $\mathrm{DHODH}$, a rate-limiting enzyme in de novo pyrimidine biosynthesis, are broad-spectrum antiviral against RNA viruses including newly emerged coronavirus SARS-CoV-2. Preprint at bioRxiv https://doi.org/ 10.1101/2020.03.11.983056 (2020).

56. Ghadiri, M. et al. Dimethyl fumarate-induced lymphopenia in MS due to differential T-cell subset apoptosis. Neurol. Neuroimmunol. Neuroinflamm 4 e340 (2017)

57. Li, R. et al. Dimethyl fumarate treatment mediates an anti-inflammatory shift in B cell subsets of patients with multiple sclerosis. J. Immunol. 198, 691-698 (2017)

58. Mehta, D. et al. Effect of dimethyl fumarate on lymphocytes in RRMS: implications for clinical practice. Neurology 92, e1724-e1738 (2019).

59. Rosenkranz, T., Novas, M. \& Terborg, C. PML in a patient with lymphocytopenia treated with dimethyl fumarate. N. Engl. J. Med. 372, 1476-1478 (2015).

60. Perini, P. et al. Herpes simplex virus encephalitis temporally associated with dimethyl fumarate-induced lymphopenia in a multiple sclerosis patient. Multiple Scler Relat Disord 26, 68-70 (2018).

61. Niino, M. et al. Natalizumab effects on immune cell responses in multiple sclerosis. Ann. Neurol. 59 748-754 (2006)

62. Butzkueven, $\mathrm{H}$. et al. Long-term safety and effectiveness of natalizumab treatment in clinical practice: 10 years of real-world data from the Tysabri Observational Program (TOP). J. Neurol. Neurosurg. Psychiatr. 91, 660-668 (2020).

63. Polman, C. H. et al. A randomized, placebo-controlled trial of natalizumab for relapsing multiple sclerosis. N. Engl. J. Med. 354, 899-910 (2006).

64. Foley, J. et al. The 5-year Tysabri Global Observationa Program in Safety (TYGRIS) study confirms the longterm safety profile of natalizumab treatment in multiple sclerosis. Multiple Scler. Relat. Disord. 39, 101863 (2019)

65. Schwab, N., Schneider-Hohendorf, T., Melzer, N. Cutter, G. \& Wiendl, H. Natalizumab-associated PML: challenges with incidence, resulting risk, and risk stratification. Neurology 88, 1197-1205 (2017).

66. Prosperini, L. et al. Post-natalizumab disease reactivation in multiple sclerosis: systematic review and meta-analysis. Ther. Adv. Neurol. Disord. https:/ doi.org/10.1177/1756286419837809 (2019).

67. Louapre, $C$ et al. Patients with MS treated with immunosuppressive agents: across the COVID-19 spectrum. Rev. Neurol. 176, 523-525 (2020).

68. Borriello, G. \& lanniello, A. COVID-19 occurring during natalizumab treatment: a case report in a patient with extended interval dosing approach. Mult. Scler. Relat. Disord. 41, 102165 (2020).

69. Ryerson, L. Z. et al. Risk of natalizumab-associated PML in patients with MS is reduced with extended interval dosing. Neurology 93, e1452-e1462 (2019).

70. Paniz-Mondolfi, A. et al. Central nervous system involvement by severe acute respiratory syndrome coronavirus-2 (SARS-CoV-2). J. Med. Virol. 92, 699-702 (2020)

71. Asadi-Pooya, A. A. \& Simani, L. Central nervous system manifestations of COVID-19: a systematic review. J. Neurol. Sci. 413, 116832 (2020).

72. Sigrist, C. J., Bridge, A. \& Le Mercier, P. A potential role for integrins in host cell entry by SARS-CoV-2. Antivir. Res. 177, 104759 (2020).

73. Tresoldi, I., Sangiuolo, C. F., Manzari, V. \& Modesti, A SARS-COV-2 and infectivity: possible increase in infectivity associated to integrin motif expression. J. Med. Virol. 177, 104759 (2020)

74. Arvin, A. M. et al. Varicella-zoster virus infections in patients treated with fingolimod: risk assessment and consensus recommendations for management. JAMA Neurol. 72, 31-39 (2015)

75. Tagawa, A. et al. Hepatitis C virus (HCV) reactivation during fingolimod treatment for relapsing and remitting multiple sclerosis. Mult. Scler. Relat. Disord. 9, 155-157 (2016)

76. Beadnall, H. N., Gill, A. J., Riminton, S. \& Barnett, M. H. Virus-related Merkel cell carcinoma complicating fingolimod treatment for multiple sclerosis. Neurology 87, 2595-2597 (2016)

77. Benedetti, M. D. et al. HPV-related papillary squamous cell carcinoma of the tonsil during treatment with fingolimod. Mult. Scler. Relat. Disord. 23, 24-26 (2018).

78. Cohen, J. A. et al. Safety and efficacy of the selective sphingosine 1-phosphate receptor modulator ozanimod in relapsing multiple sclerosis (RADIANCE): a randomised, placebo-controlled, phase 2 trial. Lancet Neurol. 15, 373-381 (2016).

79. Cohen, J. A. et al. Efficacy and safety of ozanimod in multiple sclerosis: dose-blinded extension of a randomized phase II study. Mult. Scler. 25, 1255-1262 (2019).

80. Comi, G. et al. Safety and efficacy of ozanimod versus interferon beta- $1 \mathrm{a}$ in relapsing multiple sclerosis (SUNBEAM): a multicentre, randomised, minimum 12-month, phase 3 trial. Lancet Neurol. 18 1009-1020 (2019)

81. Kappos, L. et al. Siponimod versus placebo in secondary progressive multiple sclerosis (EXPAND) a double-blind, randomised, phase 3 study. Lancet 391, 1263-1273 (2018)

82. Ghadiri, M. et al. Reconstitution of the peripheral immune repertoire following withdrawal of fingolimod. Mult. Scler. 23, 1225-1232 (2017).

83. Tay, M. Z., Poh, C. M., Rénia, L., MacAry, P. A. \& $\mathrm{Ng}$, L. F. P. The trinity of COVID-19: immunity, inflammation and intervention. Nat. Rev. Immunol. 20, 363-374 (2020)

84. US National Library of Medicine. ClinicalTrials.gov https://clinicaltrials.gov/ct2/show/NCT04280588 (2020).

85. Cooper, N. \& Arnold, D. M. The effect of rituximab on humoral and cell mediated immunity and infection in the treatment of autoimmune diseases. Br. J. Haematol. 149, 3-13 (2010).

86. Hauser, S. L. et al. Ocrelizumab versus interferon beta- $1 \mathrm{a}$ in relapsing multiple sclerosis. N. Engl. J. Med. 376, 221-234 (2017).

87. Bar-Or, A. et al. Subcutaneous ofatumumab in patients with relapsing-remitting multiple sclerosis: The MIRROR study. Neurology 90, e1805-e1814 (2018).

88. Ciardi, M. R. et al. Reactivation of hepatitis B virus with immune-escape mutations after ocrelizumab treatment for multiple sclerosis. Open. Forum Infect. Dis. 6, ofy356 (2019).

89. Lin, K.-M., Lin, J.-C., Tseng, W.-Y. \& Cheng, T.-T. Rituximab-induced hepatitis $C$ virus reactivation in rheumatoid arthritis. J. Microbiol. Immunol. Infect. 46, 65-67 (2013)
90. Sul, J. et al. Progressive multifocal leukoencephalopathy in a patient on ocrelizumab monotherapy. Neurology 94, $4875(2020)$

91. Novi, G. et al. COVID-19 in a MS patient treated with ocrelizumab: does immunosuppression have a protective role? Mult. Scler. Relat. Disord. 42, 102120 (2020).

92. Hughes, R., Pedotti, R. \& Koendgen, H. COVID-19 in persons with multiple sclerosis treated with ocrelizumab - a pharmacovigilance case series. Mult. Scler. Relat. Disord. 42, 102192 (2020).

93. Amor, S., Baker, D., Khoury, S. J., Schmierer, K. \& Giovanonni, G. SARS-CoV-2 and multiple sclerosis: not all immune depleting DMTs are equal or bad. Ann. Neurol. 87, 794-797 (2020)

94. Safavi, F., Nourbakhsh, B. \& Azimi, A. R. B-cell depleting therapies may affect susceptibility to acute respiratory illness among patients with multiple sclerosis during the early COVID-19 epidemic in Iran. Mult. Scler. Relat. Disord. 43, 102195 (2020).

95. Quinti, I. et al. A possible role for B cells in COVID-19? Lesson from patients with agammaglobulinemia. J. Allergy Clin. Immunol. https://doi.org/10.1016/j.jaci. 2020.04.013 (2020)

96. Bearden, C. M. et al. Rituximab inhibits the in vivo primary and secondary antibody response to a neoantigen, bacteriophage phiX174. Am J. Transplant. 5, 50-57 (2005)

97. Novi, G. et al. Ocrelizumab does not impair B- and T-cell responses to primary VZV infection in a patient with MS. Neurol. Neuroimmunol. Neuroinflamm. 7 e695 (2020).

98. Stokmeier, D. et al. Effect of ocrelizumab on vaccine responses in patients with multiple sclerosis [abstract] Neurology 90 (Suppl. 15), S36.002 (2018).

99. Wiendl, H. \& Kieseier, B. Reprogramming the immune repertoire with alemtuzumab in MS. Nat. Rev. Neurol. 9, 125-126 (2013).

100. Lünemann, J. D., Ruck, T., Muraro, P. A., Bar-Or, A. \& Wiendl, H. Immune reconstitution therapies: concepts for durable remission in multiple sclerosis. Nat. Rev. Neurol. 16, 56-62 (2020).

101. Möhn, N. et al. Alemtuzumab therapy changes immunoglobulin levels in peripheral blood and CSF Neurol. Neuroimmunol. Neuroinflamm. 7, e654 (2020).

102. Wray, S. et al. Infection risk with alemtuzumab decreases over time: pooled analysis of 6-year data from the CAMMS223, CARE-MS I, and CARE-MS II studies and the CAMMS03409 extension study. Mult. Scler. 25, 1605-1617 (2019).

103. Wiendl, H. et al. Lymphocyte pharmacodynamics are not associated with autoimmunity or efficacy after alemtuzumab. Neurol. Neuroimmunol. Neuroinflamm 7, e635 (2020).

104. Coles, A. J. et al. Alemtuzumab CARE-MS II 5-year follow-up: efficacy and safety findings. Neurology 89 1117-1126 (2017)

105. Giovannoni, G. Cladribine to treat relapsing forms of multiple sclerosis. Neurotherapeutics 14, 874-887 (2017).

106. Wiendl, H. Cladribine - an old newcomer for pulsed immune reconstitution in MS. Nat. Rev. Neurol. 13 573-574 (2017).

107. Baker, D. et al. Both cladribine and alemtuzumab may effect MS via B-cell depletion. Neurol. Neuroimmunol. Neuroinflamm. 4, e360 (2017).

108. Ceronie, B. et al. Cladribine treatment of multiple sclerosis is associated with depletion of memory B cells. J. Neurol. 265, 1199-1209 (2018).

109. Havrdova, E. et al. Alemtuzumab CARE-MS I 5-year follow-up: durable efficacy in the absence of continuous MS therapy. Neurology 89, 1107-1116 (2017).

110. Willis, M. D. \& Robertson, N. P. Alemtuzumab for the treatment of multiple sclerosis. Ther. Clin. Risk Manag 11, 525-534 (2015)

111. Thomas, K., Eisele, J., Rodriguez-Leal, F A , Hainke, U. $\Sigma$ Ziemssen, T. Acute effects of alemtuzumab infusion in patients with active relapsing-remitting MS. Neurol. Neuroimmunol. Neuroinflamm. 3, e228 (2016).

112. Jacobs, B. M. et al. Cladribine: mechanisms and mysteries in multiple sclerosis. J. Neurol. Neurosurg. Psychiatry 89, 1266-1271 (2018).

113. Burt, R. K. et al. Effect of nonmyeloablative hematopoietic stem cell transplantation vs continued disease-modifying therapy on disease progression in patients with relapsing-remitting multiple sclerosis: a randomized clinical trial. JAMA 321, 165-174 (2019).

114. Muraro, P. A. et al. Autologous haematopoietic stem cell transplantation for treatment of multiple sclerosis. Nat. Rev. Neurol. 13, 391-405 (2017). 
115. Zhou, F. et al. SARS-CoV-2 shedding and infectivity authors' reply. Lancet 395, 1340 (2020)

116. Gudbjartsson, D. F. et al. Spread of SARS-CoV-2 in the Icelandic population. N. Engl. J. Med. 382 2302-2315 (2020).

117. Yang, J.-R. et al. Persistent viral RNA positivity during recovery period of a patient with SARS-CoV-2 infection. J. Med. Virol. https://doi.org/10.1002/ jmv. 25940 (2020).

118. Prescott, J. et al. Pathogenicity and viral shedding of MERS-CoV in immunocompromised rhesus macaques. Front. Immunol. 9, 205 (2018).

119. de Lima, C. R. A. et al. Prolonged respiratory viral shedding in transplant patients. Transpl. Infect. Dis. 16, 165-169 (2014)

120. Nowak, M. D., Sordillo, E. M., Gitman, M. R. $\delta$ Paniz Mondolfi, A. E. Co-infection in SARS-CoV-2 infected patients: where are influenza virus and rhinovirus/enterovirus? J. Med. Virol. https://doi. org/10.1002/jmv.25953 (2020).

121. Rawson, T. M. et al. Bacterial and fungal co-infection in individuals with coronavirus: a rapid review to support COVID-19 antimicrobial prescribing Clin. Infect. Dis. https://doi.org/10.1093/cid/ciaa530 (2020).

122. Olberg, H. K. et al. Antibody response to seasonal influenza vaccination in patients with multiple sclerosis receiving immunomodulatory therapy. Eur. J. Neurol. 25, 527-534 (2018)

123. Metze, C. et al. Immunogenicity and predictors of response to a single dose trivalent seasonal influenza vaccine in multiple sclerosis patients receiving disease-modifying therapies. CNS Neurosci. Ther. 25 245-254 (2019)

124. Klotz, L. et al. Risks and risk management in modern multiple sclerosis immunotherapeutic treatment. Ther. Adv. Neurol. Disord. 12, 1756286419836571 (2019).

125. Koyama, S., Ishii, K. J., Coban, C. \& Akira, S. Innate immune response to viral infection. Cytokine 43 , 336-341 (2008)

126. Cao, X. COVID-19: immunopathology and its implications for therapy. Nat. Rev. Immunol. $\mathbf{2 0}$ 269-270 (2020)

127. Mehta, P. et al. COVID-19: consider cytokine storm syndromes and immunosuppression. Lancet 395 , 1033-1034 (2020).

128. Berger, J. R., Brandstadter, R. \& Bar-Or, A COVID-19 and MS disease-modifying therapies. Neurol. Neuroimmunol. Neuroinflamm. 7, e76 (2020).

129. Zhang, B. et al. Immune phenotyping based on neutrophil-to-lymphocyte ratio and IgG predicts disease severity and outcome for patients with COVID-19. Preprint at medrxiv.org https://doi.org/ 10.1101/2020.03.12.20035048 (2020).

130. Sarzi-Puttini, P et al. COVID-19, cytokines and immunosuppression: what can we learn from severe acute respiratory syndrome? Clin. Exp. Rheumatol. 38, 337-342 (2020)

131. Napolitano, M., Fabbrocini, G. \& Patruno, C. Potential role of Janus kinase inhibitors in COVID-19. J. Am. Acad. Dermatol. 83, e65 (2020).

132. Risitano, A. M. et al. Complement as a target in COVID-19? Nat. Rev. Immunol. 20, 343-344 (2020).

133. Mastaglio, S. et al. The first case of COVID-19 treated with the complement C3 inhibitor AMY-101. Clin. Immunol. 215, 108450 (2020).

134. Diurno, F. et al. Eculizumab treatment in patients with COVID-19: preliminary results from real life ASL Napoli 2 Nord experience. Eur. Rev. Med. Pharmacol. Sci. 24, 4040-4047 (2020).

135. Seminari, E. et al. SARS Cov2 infection in a renal transplanted patient: a case report. Am. J. Transplant. https://doi.org/10.1111/ajt.15902 (2020).

136. Norsa, L. et al. Uneventful course in IBD patients during severe acute respiratory syndrome coronovirus 2 outbreak in northern Italy. Gastroenterology https://doi.org/10.1053/j.gastro.2020.03.062 (2020).

137. Romanelli, A. \& Mascolo, S. Immunosuppression drug-related and clinical manifestation of coronavirus disease 2019: a therapeutical hypothesis. Am. J. Transplant. https://doi.org/10.1111/ajt.15905 (2020).

138. Guan, W.-J. et al. Clinical characteristics of coronavirus disease 2019 in China. N. Engl. J. Med. 382 , 1708-1720 (2020).

Acknowledgements

The authors thank E. East for native language editing.

\section{Author contributions}

C.K. and H.W. researched data for the article and wrote the article. All authors made substantial contributions to discussion of the content and reviewed and edited the manuscript before submission.

\section{Competing interests}

C.K. has received travel support and/or speaking honoraria from Biogen, Sanofi Genzyme, Roche, Merck and Teva within the past years. S.R. has received funds within the past 5 years for (but not limited to) travel support, honoraria, trial payments, research and clinical support to the neurology department of which he is a member from several bodies and charities such as Lambert Initiative, Beeren Foundation and anonymous donors, and from Baxter, Bayer Schering, Biogen Idec, CSL, Sanofi Genzyme, Grifols, Octapharma, Merck, Novartis, Roche, Sanofi Aventis Genzyme, Servier and Teva. S.R. also declares the following competing interests: co-founder and shareholder of Medical Safety Systems trading as RxMx (including grant and/or contracts with Genzyme Novartis, Roche and Janssen); receives payment for contributions to the National IVIg Governance Advisory Council $\delta$ Specialist Working Group Australia (Neurology) and the Australian Medical Services Advisory Committee ad hoc sub-committee on IVIg; unpaid member of the Australian Technical Advisory Group on Immunization Varicella Zoster working party; receives a public salary as a staff specialist neurologist from Concord Hospital Sydney Local Health District; receives private billings from patients and Medicare Australia reimbursement as a private practice neurologist; and is an unpaid medical adviser to various patient and advocacy groups. A.B.-O. serves on scientific advisory boards for Atara Biotherapeutics, Biogen Idec, Celgene/Receptos, Janssen/Actelion, Merck/EMD Serono, Novartis, Roche/ Genentech and Sanofi Genzyme, and has sponsored research agreements with Biogen Idec, Novartis and Roche/Genentech. H.W. receives honoraria for acting as a member of scientific advisory boards for Biogen, Evgen, Genzyme, MedDay Pharmaceuticals, Merck Serono, Novartis, Roche Pharma AG and Sanofi-Aventis, as well as speaker honoraria and travel support from Alexion, Biogen, Cognomed, F. Hoffmann-La Roche, Gemeinnützige Hertie-Stiftung, Merck Serono, Novartis, Roche Pharma AG, Genzyme, Teva and WebMD Global. H.W. is also a paid consultant for Abbvie, Actelion, Biogen, IGES, Johnson \& Johnson, Merck, Novartis, Roche and Sanofi. His research is funded by the German Ministry for Education and Research (BMBF), Deutsche Forschungsgemeinschaft (DFG), Else Kröner Fresenius Foundation, Fresenius Foundation, the European Union, Hertie Foundation, NRW Ministry of Education and Research, Interdisciplinary Center for Clinical Studies (IZKF) Muenster and RE Children's Foundation, Biogen, GlaxoSmithKline $\mathrm{GmbH}$, Roche Pharma AG and Sanofi Genzyme.

\section{Peer review information}

Nature Reviews Neurology thanks G. Giovannoni, S. Pittock, V.W. Yong and the other, anonymous, reviewer(s) for their contribution to the peer review of this work.

\section{Publisher's note}

Springer Nature remains neutral with regard to jurisdictional claims in published maps and institutional affiliations.

\section{Supplementary information}

Supplementary information is available for this paper at https://doi.org/10.1038/s41582-020-0385-8.

\section{RELATED LINKS}

Coronavirus and MS reporting database (COVIMS):

https://www.covims.org

COVID-19 Associated Risks and Effects in Myasthenia

Gravis (CARE-MG): https://myasthenia.org/For-Professionals/

Resources-for-Professionals/CARE-MC

Lean European Open Survey on SARS-CoV-2 Infected

Patients (LEOSS): https://leoss.net

Ongoing clinical trials of corticosteroids in COVID-19:

https://clinicaltrials.gov/ct2/results?cond=covid +Eterm=

corticosteroids\&cntry $=\&$ state $=\delta c i t y=\& d i s t=$

C) Springer Nature Limited 2020 